\title{
High Risk Acute Leukemia
}

National Cancer Institute

\section{Source}

National Cancer Institute. High Risk Acute Leukemia. NCI Thesaurus. Code C122458.

Acute leukemia patients are stratified into the high risk group when their minimal residual disease levels are higher than $0.05 \%$. 